\title{
Comparison of individual and pooled urine samples for estimating the presence and intensity of Schistosoma haematobium infections at the population level
}

\author{
Abraham Degarege ${ }^{1,2^{*}}$ D, Berhanu Erko ${ }^{1}, Z^{2}$ Zeleke Mekonnen ${ }^{3}$, Mengistu Legesse $^{1}$, Yohannes Negash$^{1}$,
} Jozef Vercruysse ${ }^{4}$ and Bruno Levecke ${ }^{4}$

\begin{abstract}
Background: There is a lack of cost-effective diagnostic strategies to evaluate whether mass drug administration (MDA) programmes to control Schistosoma haematobium progress as anticipated. The purpose of this study is to provide a proof-of-principle for examination of pooled urine samples as a strategy for rapid assessment of presence and intensity of Schistosoma haematobium infections at the population level.
\end{abstract}

Methods: A total of 640 urine samples were collected from 520 school-aged children (520 at baseline and 120 at follow-up) during a clinical trial that was designed to assess the efficacy of praziquantel against Schistosoma haematobium infections in Ethiopia. Individual and pooled urine samples were screened using the filtration technique (volume of $10 \mathrm{ml}$ urine) to determine the number of $\mathrm{S}$. haematobium eggs in $10 \mathrm{ml}$ of urine. Samples were pooled into pools of $5(n=128), 10(n=64)$ and $20(n=32)$ individual samples. The sensitivity, the probability of finding at least one egg in a pooled sample when the mean urine egg count (UEC) of the corresponding individual urine samples was not zero, was calculated for each pool size. UECs of a pooled examination strategy were compared with the mean UECs of the corresponding individual samples.

Results: The sensitivity of a pooled examination strategy was $50.6 \%$ for pools of 5, 68.6 \% for pools of 10 and $63.3 \%$ for pools of 20 . The sensitivity of a pooled examination strategy increased as a function of increasing mean UEC of the corresponding individual urine samples. For each of the three pool sizes, there was a significant positive correlation between mean UECs of individual and those obtained in pooled samples (correlation coefficient: 0.81 - 0.93). Examination of pools of 5 provided significantly lower UECs compared to the individual examination strategy (3.9 eggs/10 $\mathrm{ml}$ urine versus $5.0 \mathrm{eggs} / 10 \mathrm{ml}$ urine). For pools of $10(4.4 \mathrm{eggs} / 10 \mathrm{ml}$ ) and 20 (4.2 eggs/10 ml), no significant difference in UECs was observed.

Conclusions: Examination of pooled urine samples applying urine filtration holds promise for rapid assessment of intensity of $S$. haematobium infections, but may fail to detect presence of infections when endemicity is low. Further investigation is required to determine when and how pooling can be optimally implemented in monitoring of mass drug administration programmes.

Keywords: Schistosoma haematobium, Pooling, Infection intensity, Sensitivity, Mass drug administration, Monitoring and evaluation, Ethiopia

\footnotetext{
* Correspondence: abrahamdegarege@yahoo.com

'Aklilu Lemma Institute of Pathobiology, Addis Ababa University, Addis

Ababa, Ethiopia

${ }^{2}$ Department of Epidemiology, Robert Stempel College of Public Health and

Social Work, Florida International University, Miami, FL, USA

Full list of author information is available at the end of the article
}

\section{Ciomed Central}

(c) 2015 Degarege et al. Open Access This article is distributed under the terms of the Creative Commons Attribution 4.0 International License (http://creativecommons.org/licenses/by/4.0/), which permits unrestricted use, distribution, and reproduction in any medium, provided you give appropriate credit to the original author(s) and the source, provide a link to the Creative Commons license, and indicate if changes were made. The Creative Commons Public Domain Dedication waiver (http://creativecommons.org/publicdomain/zero/1.0/) applies to the data made available in this article, unless otherwise stated. 


\section{Background}

Neglected tropical diseases (NTDs), which are caused by a variety of viruses, bacteria and parasites, pose an important burden on public health in several parts of the world. NTDs are the fourth most important group contributing to the global burden attributable to communicable diseases, accounting for $\sim 48$ million disabilityadjusted life years lost each year, mainly affecting people in developing countries $[1,2]$.

Recently, the World Health Organization (WHO) has set the ambitious targets to control, eliminate and eradicate 10 specific NTDs by 2020, including urinary schistosomiasis [3]. These targets were subsequently endorsed in the London Declaration on NTDs (January, 2012), and supported by more than 70 pharmaceutical companies, governments and global health organizations by sustaining or expanding NTD drug donation programmes. For example, the number of tablets donated for the control of schistosmiassis from Merck KGaA company increased from $\sim 20$ million tablets in 2012 to 250 million annually for an unlimited period [4]. Since the London Declaration, more than 5.5 billion tablets have been donated [5].

Although this is probably the largest public health drug donation programme in the world, it also creates the need to adequately monitor these mass drug administration (MDA) programmes to verify whether the targets set are being met, and if necessary, to adjust the programme implemented. Currently, the impact of MDA programmes is evaluated by re-assessing the epidemiology (prevalence and intensity of infections for some NTDs) through largescale epidemiological surveys in which subjects are individually screened. However, this strategy of individually screening a large number of subjects faces some important financial and technical obstacles, particularly when MDA programmes are mainly operating in resource-limited settings. In veterinary medicine, pooling samples has been applied to reduce number of diagnosis, and hence reducing the costs for monitoring the impact of measures to control helminth infections [6-8]. Recently, pooling stool samples has also been evaluated for the assessment of two NTDs, including soil-transmitted helminthiasis and schistosomiasis (Schistosoma mansoni) $[9,10]$. The results indicated that examination of pooled stool samples did not compromise on the accuracy of the assessment of the intensity of soil-transmitted helminth and Schistosoma mansoni infections, while reducing the time in the laboratory by at least $70 \%$ [10]. These findings suggest that pooling samples could result in important cost-savings in large-scale epidemiological surveys. This is particularly when these NTDs are often heterogeneously scattered across large geographical areas [11]. Today, it remains unclear whether pooling would also be a reliable strategy to assess other NTDs that are diagnosed in other specimens. The purpose of this study is to provide a proof-of- principle for examination of pooled urine samples as a strategy for rapid assessment of the presence and the intensity of $S$. haematobium infections at the population level.

\section{Methods}

\section{Study area and population}

The study was conducted in Middle Awash Valley of the Afar Region (northeastern Ethiopia) between February and May 2014, including four Afar ethnic villages (Anbesh, Buri, Hassabo and Hanledebe) of the Amibara District. These villages are located 300 to $350 \mathrm{~km}$ from the capital Addis Ababa at an altitude of approximately $725 \mathrm{~m}$ above sea level. The annual rainfall is on average $654 \mathrm{~mm}^{3}$, the average temperature is $25.6^{\circ} \mathrm{C}$. Previous studies in Buri and Hassabo villages reported a prevalence of urinary schistosomiasis ranging from 24.5 to $47.6 \%$ in children $[12,13]$. The current study focused on school-aged children, all children between 5 and 16 years of age being eligible for the study.

\section{Study design}

This study was part of a multi-country trial designed to assess the efficacy of a single dose of praziquantel $(40 \mathrm{mg} / \mathrm{kg})$ against Schistosoma spp. Infections in school-aged children, including Schistosoma mansoni (Cameroon, Ethiopia, Mali, and Tanzania), S. haematobium (Cameroon, Ethiopia, Mali, and Tanzania) and S. japonicum (the Philipines). For this trial, the initial aim was to enroll, at least 125 infected children per Schistosoma species at each study site. As a secondary objective, the proof-of-principle for examination of pooled urine samples as a strategy for rapid assessment of presence and intensity of S. haematobium infections was evaluated in Ethiopia. To this end, eggs of S. haematobium eggs were quantified in urine samples collected during the pre-intervention and post-intervention applying both an individual and a pooled examination strategy. In the past, pools of 10, 20 and 60 have been applied for quantifying eggs in soil-transmitted helminthiasis in stool. However, due to a more focal distribution of schistosomiasis, which may result in more false negative test results when too many samples are pooled, we opted for pooling into pools of 5,10 and 20 [10].

\section{Field and laboratory procedures}

The headmasters of the schools and village administrators were informed about the aim of the study. Children, who were not attending school, were brought to the school by the village administrator. Children who agreed to participate in the study were asked to bring their parents to the school. The field team, the head master and the village administrators then explained the purpose of the study in more detail to both the children and the parents. Only those children who were willing to 
participate and whose parents approved their participation in the study were given labeled plastic containers $(200 \mathrm{ml}$ capacity) in order to provide urine samples. Urine samples were collected between 10:00 am and 2:00 pm. For each child, two vials containing 0.1 formalin (37 \% formaldehyde) were subsequently filled with $10 \mathrm{ml}$ urine. The first vial was individually processed applying the urine filtration technique one week after sample collection [14]. For the filtration of urine we used polycarbonate membrane filters of $13 \mathrm{~mm}$ diameter and 12 to $14 \mu \mathrm{m}$ pore size (Sterlitech, Kent, WA, United States of America)), the second vial was used to make pools of urine.

Urine samples were pooled by adapting a cascade system that had been previously described for stool samples [9, a visual tutorial is available at https://www.youtube.com/ watch?v=IUZijtBABn0)]. In the past, pools of 10, 20 and 60 have been applied for quantifying eggs of soil-transmitted helminths in stool samples [9]. However, due to a more focal distribution of schistosomiasis, which may result in more false negative test results when too many samples are pooled, we opted to pool individual urine samples into pools of 5, 10 and 20. Figure 1 describes the procedure to pool urine samples. Pools of 5, 10 and 20 individual samples were made applying the following procedures. First, plastic vials containing urine samples in rows of 5 samples were arranged according to the unique subject identifier (increasing order). Assuming that there is no correlation between this subject identifier and the urine egg counts (UECs), we assumed that the samples were randomly arranged. Then, $10 \mathrm{ml}$ from each vial of the same row was transferred to a $50 \mathrm{ml}$ vial to prepare pools of 5 individual samples. Afterwards, $25 \mathrm{ml}$ urine from each of two different vials containing pooled samples of 5 individuals was transferred (after shaking) to another $50 \mathrm{ml}$ vial to produce pools of 10 individual samples. Finally, $25 \mathrm{ml}$ urine from each of two different vials containing pooled samples of 10 individuals was transferred (after shaking) to another $50 \mathrm{ml}$ size vial to produce pools of 20 individual samples. Finally, $10 \mathrm{ml}$ of each of the pooled urine samples was filtered and examined using the same procedure as used for individual samples.

Fourteen to 21 days post-intervention, urine samples from subjects excreting eggs at the pre-intervention survey were again collected and processed applying the same procedures during the pre-intervention survey. Eggs of S. haematobium were quantified in $10 \mathrm{ml}$ of both individual and pooled urine samples within 1 week after collecting the samples applying the urine filtration technique.

\section{Statistical data analysis}

We compared both the qualitative (sensitivity) and the quantitative (UECs) diagnostic performance of a pooled examination strategy at the pooled level. Sensitivity, the probability of finding at least one egg in a pooled sample when the mean UECs of the corresponding individual urine samples was not zero, was calculated for each pool size. To this end, we assumed a specificity of $100 \%$ for both strategies. We explored the variation in sensitivity for each pool size by a logistic regression model. To do this, we set the pooled test result (positive/negative) as the outcome, and the mean UECs of the corresponding individual urine samples as covariate. The predictive power of these models was evaluated by the proportion of the observed outcome that was correctly predicted by the model. For this, an individual probability $>0.5$ was set as a positive test result, and negative if different. Finally, the sensitivity for each of the observed values of mean UECs was calculated based on these models.

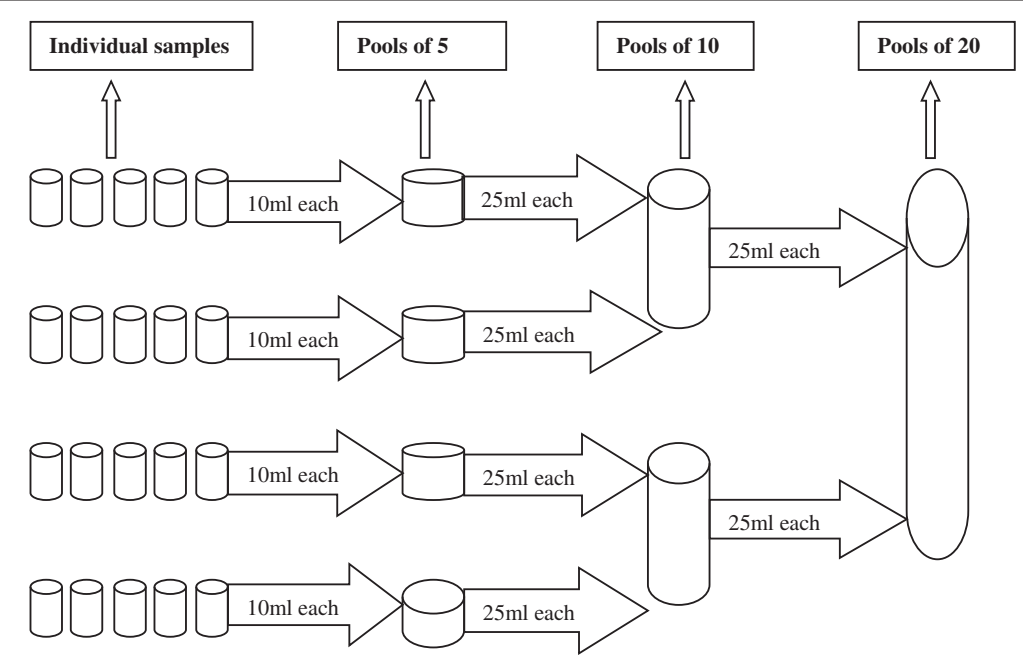

Fig. 1 The procedure to obtain pools of 5, 10 and 20 individual samples 
The agreement in UECs across the three methods was verified by a permutation test (10,000 iterations) based on Pearson correlation coefficient and differences in UECs. The Tukey's method was applied for pairwise comparison. The level of significance was set at $p<0.05$.

\section{Ethical consideration}

The study was approved by the Institutional Review Board of Aklilu Lemma Institute of Pathobiology, Addis Ababa University (Ref. No. IRB/22-A/2012/13). The District Health Office, school authorities, teachers, parents, and the children were informed about the purpose and procedures of the study. Only those children who were willing to participate and from whom the parents or guardian gave verbal informed consent were included in the study. As the study population was mainly illiterate the Institutional Review Board of Aklilu Lemma Institute of Pathobiology indorsed oral consenting of the parents or guardians of the children. Since the study involved minimal risk the Institutional Review Board did not require tape recording or any other form of proof of the informed consent processes. Children who were infected with $S$. haematobium infection were treated with praziquantel (40 $\mathrm{mg} / \mathrm{kg}$ body weight).

\section{Results}

\section{Prevalence and infection intensity}

A total of 632 children (mean age $=9.8$ years, female $/$ male ratio $=0.72$ ) at the pre-intervention survey and 151 children during the post-intervention were screened individually for S. haematobium infections (Fig. 2). A total of 640 samples, including 520 samples at pre-intervention and 120 samples at post-intervention, were also analysed using the pooling strategy. When samples were individually examined, S. haematobium eggs were found in 181 out of 640 urine samples $(28.3 \%)$. The number of positive pools was 84/128 (65.6\%), 51/64 (79.7\%) and 30/32 (93.6\%) for pools of 5, 10 and 20 individual stool samples, respectively. The arithmetic mean of UECs equaled $5.0 \mathrm{eggs} / 10 \mathrm{ml}$, with individual UECs ranging from 0 to 426 eggs $/ 10 \mathrm{ml}$. All the 120 samples at postintervention were negative for $S$. haematobium eggs. The arithmetic mean UEC of the 640 samples based on examination of pools of 5 , pools of 10 and pools of 20 was 3.9 eggs/10 ml urine, 4.4 eggs/10 $\mathrm{ml}$ and 4.2 eggs/10 ml, respectively.

\section{Sensitivity}

Eggs were not found in pools for which the mean of individual UECs equaled zero (i.e. sensitivity of the individual examination strategy equaled $100 \%$ ). The sensitivity of a pooled examination strategy is summarized in Table 1. The pooled examination strategy was significantly less sensitive (pools of $5=53.9 \%, 95 \%$ confidence intervals (CI) [40.0, 61.2]; pools of $10=68.7 \%, 95 \% \mathrm{CI}$ [55.9, 81.4]; pools of $20=63.3 \%, 95 \%$ CI [46.1, 80.6]). However, the sensitivity of the pooling examination strategy increased significantly as a function of the mean of UECs of the corresponding individual samples. The pooled examination strategy was equally sensitive with the individual examination strategy when the UECs became high (Fig. 3). The models correctly predicted the observed test results in $72.9 \%$ for pools of 5 , $78.4 \%$ pools of 10 and $83.3 \%$ for pools of 20 .

\section{Infection intensity}

Overall, there was a positive significant correlation between the UECs obtained by examining pooled samples and the mean UECs of the corresponding individual urine samples (Fig. 4). The correlation coefficient (R) was the lowest for pools of $20(\mathrm{R}=0.81, p<0.001)$, and the highest for pools of $5(\mathrm{R}=0.93, p<0.001)$. Examination of pools of 5 provided significantly lower UECs compared to an individual examination strategy (mean difference $=-1.08 \mathrm{eggs} / 10 \mathrm{ml}, 95 \% \mathrm{CI}[-2.2,-0.15])$. For pools of 10 (mean difference $=-0.61$ eggs $/ 10 \mathrm{ml}$; $95 \%$ CI $[-2.2,0.79]$ and 20 (mean difference $=-0.85$ eggs/10 ml; $95 \%$ CI $[-2.92,0.77])$, no significant difference in UECs was observed (Table 2).

\section{Discussion}

To date there are no cost-effective tools to verify whether MDA programmes progress as anticipated, and if necessary, to adjust the programme implemented. The present study provides a proof-of-principle for examination of pooled urine samples as a strategy for rapid assessment of $S$. haematobium infections, by evaluating the diagnostic performance of pooling urine for both the qualitative (presence of infection) and quantitative assessment (infection intensity) of S. haematobium infections at the population level. In the current study, the sensitivity of a pooled examination strategy was low $(50.6 \%-68.6 \%)$, but increased as a function of increasing mean UEC of the corresponding individual urine samples. For each of the three pool sizes, there was a significant positive correlation between mean UECs of individual and those obtained in pooled samples (correlation coefficient: 0.81 - 0.93). A significant difference in UEC was observed only for pools of 5, resulting in significantly lower UECs compared to the individual examination strategy (3.9 eggs $/ 10 \mathrm{ml}$ urine versus 5.0 eggs $/ 10 \mathrm{ml}$ urine).

These results indicate that pooling urine samples lacks sensitivity to detect low levels of infection intensity (1$49 \mathrm{egg} / 10 \mathrm{ml}$ urine, Fig. 4), and hence applying this strategy may result in falsely declaring a population free of disease where endemicity is low. This observation is not unexpected, and can be partially explained by a 


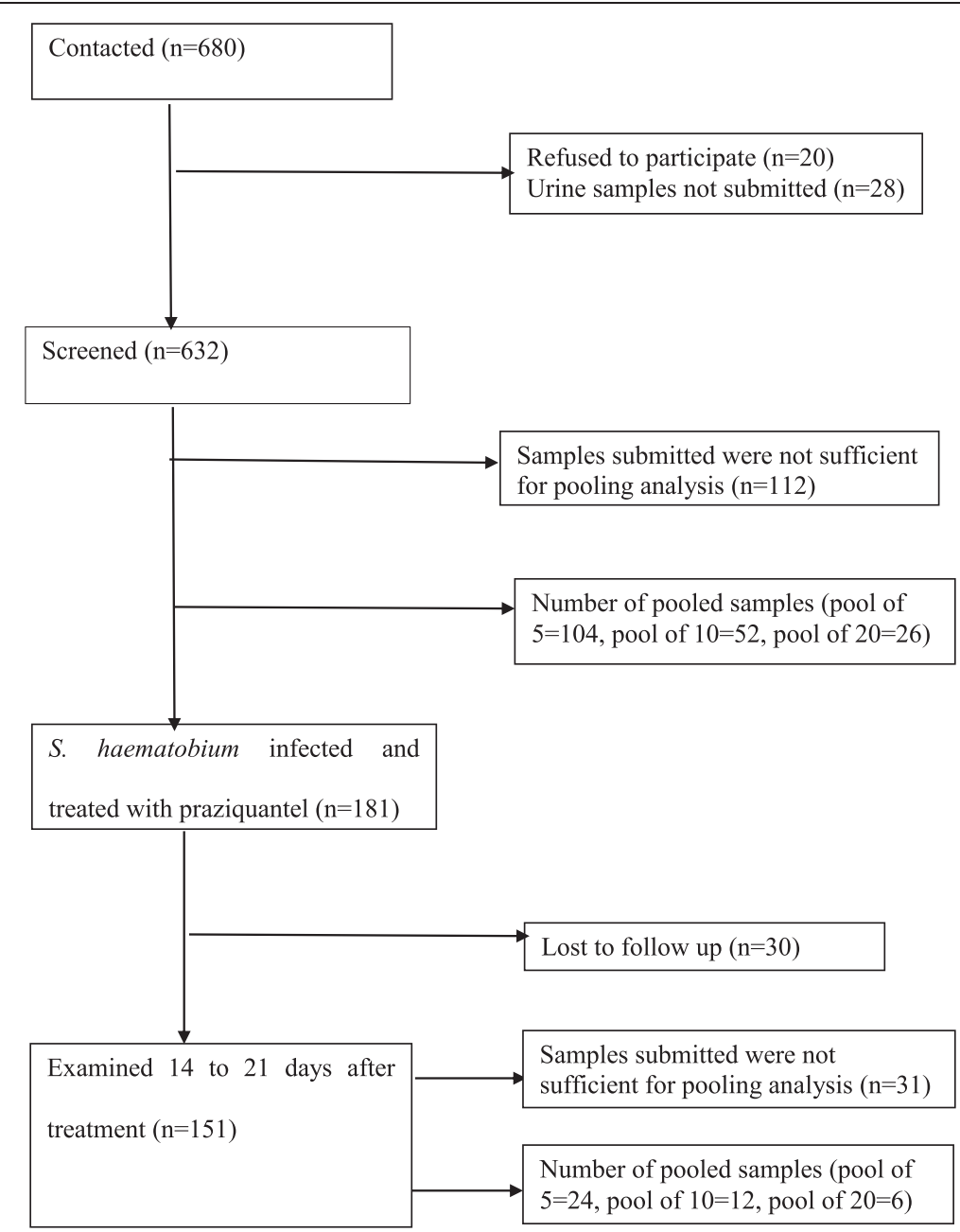

Fig. 2 Number of the study subjects enrolled, and number of pooled samples included in the analysis

combination of a dilution effect when pooling samples and the intrinsic lack of sensitivity of urine filtration to detect low egg counts $[15,16]$. To further minimize the probability of falsely declaring a population free of disease one may either consider examining more pooled urine (i.e. filtering $30 \mathrm{ml}$ of pooled urine instead of $10 \mathrm{ml}$ ) or applying a more sensitive method, such as $S$. haematobium DNA or antigen detection methods $[15,16]$. For example, in this setting it is expected that examining $30 \mathrm{ml}$ of a pool of 20 individual samples instead of $10 \mathrm{ml}$

Table 1 The number of positive Schistosoma haematobium samples and the sensitivity of the different pool sizes

\begin{tabular}{llll}
\hline Pool size & Sample size & $\begin{array}{l}\text { Number of positive } \\
\text { samples }(\%)\end{array}$ & $\begin{array}{l}\text { Sensitivity }(\%) \\
(95 \% \mathrm{Cl})\end{array}$ \\
\hline 1 & 640 & $181(28.3 \%)$ & $100 \%$ \\
5 & 128 & $84(65.6)$ & $50.6(40.0 ; 61.2)$ \\
10 & 64 & $51(79.7)$ & $68.6(55.9 ; 81.4)$ \\
20 & 32 & $30(93.6)$ & $63.3(46.1 ; 80.1)$ \\
\hline
\end{tabular}

would already increase the sensitivity from 63.3 to $95.1 \%\left(=1-(1-0.633)^{3}\right)$. The quantitative performance highlights that pooling provides comparable estimates of infection intensity, and this finding is in line with previous studies assessing pooling of stool samples as an alternative strategy to evaluate helminth infections in both animals [6-8] and humans [9, 10], highlighting that pooling of urine samples could translate into important cost-savings in large-scaled epidemiological surveys required to monitor the progress of MDA programmes.

However, there are a few aspects that require further attention for S. haematobium. First, we only applied one diagnostic method and the sample size from the different villages was not sufficient to make powerful analysis for each area separately. Complementary field and/or in silicon studies evaluating pooling of urine samples in varying scenarios of endemicity of S. haematobium, and both diagnostic (the number of samples pooled, the volume of urine examined and the sensitivity of the diagnostic technique) and sampling efforts (number of urine 


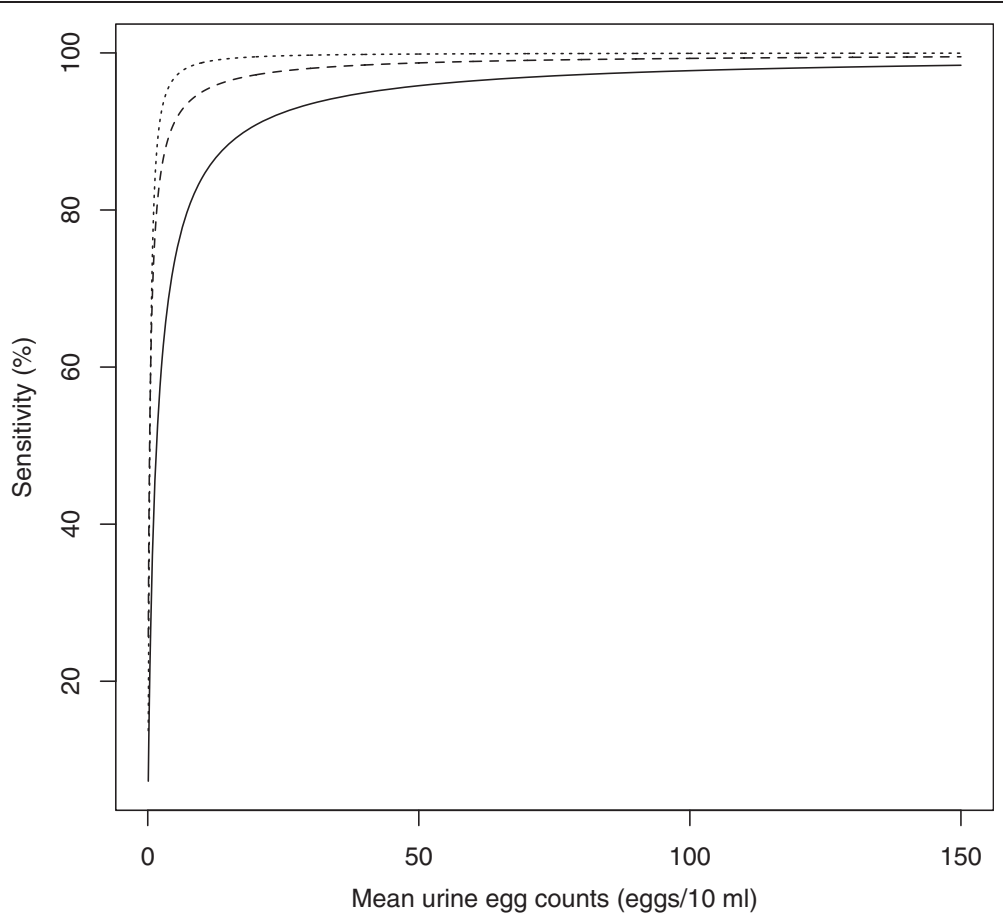

Fig. 3 The predicted sensitivity derived from logistic regression for different pool sizes. Pools of 5 samples are represented by the straight line, pools of 10 samples by the dashed line, and pools of 20 samples by the dotted line

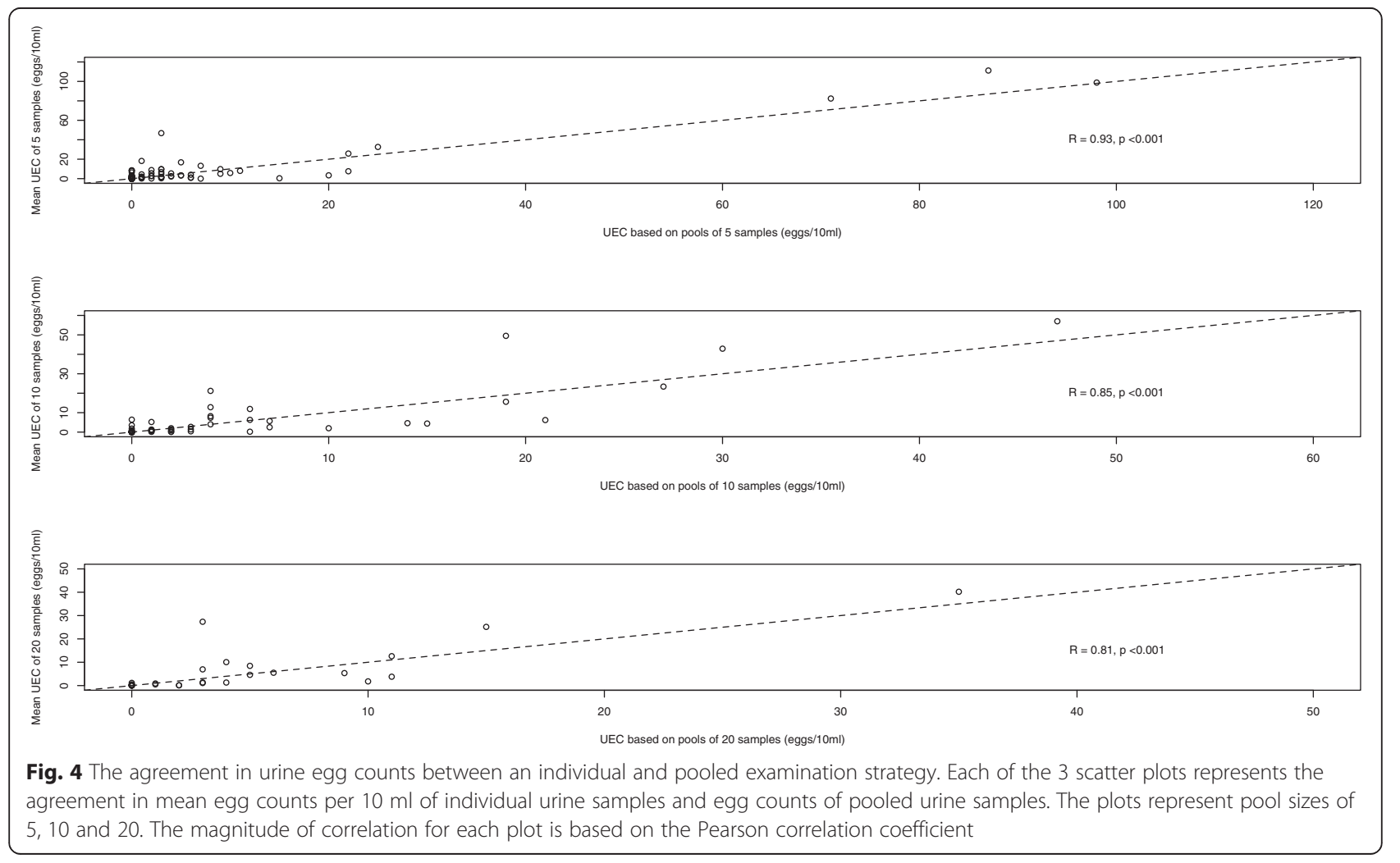


Table 2 Pair-wise comparison of arithmetic mean urine egg count between individual and a pooled examination strategy

\begin{tabular}{llll}
\hline $\begin{array}{l}\text { Pool } \\
\text { size }\end{array}$ & $\begin{array}{l}\text { Sample } \\
\text { size }\end{array}$ & $\begin{array}{l}\text { Mean UEC (eggs/10 ml) } \\
(95 \% \mathrm{Cl})\end{array}$ & $\begin{array}{l}\text { Level of significance for } \\
\text { pair-wise comparison }\end{array}$ \\
\hline 1 & 640 & $5.0(3.2 ; 7.2)$ & - \\
5 & 128 & $3.9(1.9 ; 6.5)$ & 0.032 \\
10 & 64 & $4.4(2.5 ; 6.7)$ & 0.44 \\
20 & 32 & $4.2(2.2 ; 6.8)$ & 0.44 \\
\hline
\end{tabular}

UEC: urine egg count

samples examined per subject) are required to decide when and how to apply pooling of urine samples. For example, a mathematical framework for soil-transmitted helminths has recently been developed allowing health-care decision makers to adapt their survey design according to local epidemiology (level of aggregation and intensity of worm infections) and both diagnostic and sampling effort [17, an online tool for this framework can be found at https://paradesign.shinyapps.io/paradesign/].

Second, pools were made through a cascade system (pools of 20 were made out of pools of 10 , pools of 10 were made out of pools of 5 , and pools of 5 were made out of individual samples). Although this allowed evaluating different pools sizes with a minimum of amount of urine per individual $(10 \mathrm{ml})$, it is not recommended when applied in a MDA programme. It would have major logistical issues, particularly when the number of samples to be pooled is $\geq 10$ and the number of pools is large. In addition, it may introduce bias in UEC results as pools of 10 or 20 are not entirely independent of the pools of 5 , and as the contribution of individual urine samples to the pools reduces as a function of pool size (the total volume of the pools was $50 \mathrm{ml}$ across the pool sizes, and hence the contribution of the individual samples is $10 \mathrm{ml}$ for pools of $5,5 \mathrm{ml}$ for pools of 10 and $2.5 \mathrm{ml}$ for pools of 20). This difference in contribution of individual samples to the pool may explain the current result, where the correlation between UECs obtained by examining pooled samples and the mean UEC of corresponding individual samples was lowest for pools of 20 and highest for pools of 5. To avoid this potential bias in UECs it would be recommended to pool fixed volumes of individual samples for each pool size as we did for pools of 5 rather than using pools of 5 to make pools of 10 and pools of 10 to make pools of 20 .

Third, from Table 1 it is clear that the proportion of pools containing eggs increases as a function of the number of samples pooled, and hence do not provide an accurate estimate of true underlying prevalence. Various researchers have developed statistical methods to estimate the true underlying prevalence based on the examination of pooled samples, but these need to be further validated for S. haematobium [17-19].
Finally, a cost-benefit analysis is highly recommended to verify whether pooling of urine samples in a large-scale epidemiological survey of $S$. haematobium infection is indeed cost-saving. This will help to guide programme managers and healthcare decision-makers in designing the most cost-effective survey to monitor MDA programmes aimed in the control of S. haematobium infection. To this end, cost estimates of preparing and analysing pooled urine samples should be assessed [10].

\section{Conclusions}

To conclude, examination of pooled urine samples applying urine filtration holds promise for rapid assessment of intensity of $S$. haematobium infections, but may fail to detect presence of infections when endemicity is low. Further research is required to recommend when (different levels of endemicity; different phases of programme: control $v s$. elimination) and how (number of samples collected per subject, number of samples examined per pool, the number of individual samples pooled, the volume of urine examined and the sensitivity of the diagnostic technique) pooling urine samples could translate into important cost-savings in large-scaled epidemiological surveys to monitor progress of MDA programmes.

\section{Competing interests}

The authors declare that they have no competing interests.

\section{Authors' contribution}

$A D, B E, Z M, J V, B L$ : conceived and designed the study. AD, BE, ML, YN: collected data. $A D$ and $B L$ : analysed data and drafted the manuscript. All authors read and approved the final manuscript.

\section{Acknowledgment}

We thank the staff of the Amibara district health office in the Afar region for facilitating the data collection process. We thank also the staff of the different schools and administrators in the district for assisting during data collection.

\section{Author details}

${ }^{1}$ Aklilu Lemma Institute of Pathobiology, Addis Ababa University, Addis Ababa, Ethiopia. ${ }^{2}$ Department of Epidemiology, Robert Stempel College of Public Health and Social Work, Florida International University, Miami, FL, USA. ${ }^{3}$ Department of Medical Laboratory Sciences and Pathology, College of Public Health and Medical Sciences, Jimma University, Jimma, Ethiopia. ${ }^{4}$ Department of Virology, Parasitology and Immunology, Faculty of Veterinary Medicine, Ghent University, Merelbeke, Belgium.

Received: 16 June 2015 Accepted: 10 November 2015

Published online: 16 November 2015

\section{References}

1. Hotez PJ, Alvarado M, Basáñez M-G, Bolliger I, Bourne R, Boussinesq M, et al. The Global Burden of Disease Study 2010: Interpretation and Implications for the Neglected Tropical Diseases. PLoS Negl Trop Dis. 2014;8(7):e2865.

2. Murray CJ, Vos T, Lozano R, Naghavi M, Flaxman AD, Michaud C, et al. Disability-adjusted life years (DALYs) for 291 diseases and injuries in 21 regions, 1990-2010: a systematic analysis for the Global Burden of Disease Study 2010. Lancet. 2012;380(9859):2197-223.

3. WHO. Accelerating work to overcome the global impact of neglected tropical diseases - A roadmap for implementation. Geneva: World Health Organization; 2012. http://www.who.int/neglected_diseases/NTD_ RoadMap_2012_Fullversion.pdf. Accessed September 21, 2015. 
4. WHO. Neglected tropical diseases: New agreement expands access to schistosomiasis treatment for millions. http://www.who.int/neglected_diseases/ schistosomiasis_Merck_2013/en/. Accessed June 13, 2015.

5. United to Combat Neglected Tropical Diseases. The third report: country leadership and collaboration on NTDS. http://unitingtocombatntds.org/report/ country-leadership-and-collaboration-on-NTDs. Accessed September 21, 2015.

6. FC B, Lyndal-Murphy M, Pearse B. An assessment of a composite sampling method for counting strongyled eggs in sheep faeces. Aust Vet J. 1990;67(5):165-7.

7. Mp W, Lyndal-Murphy M, Baldock FC. Evaluation of a composite method for counting helminth eggs in cattle faeces. Vet Parasitol. 1997;73(1-2):181-7.

8. Eysker M, Bakker J, van den Berg M, van Doorn DCK, Ploeger HW. The use of age-clustered pooled faecal samples for monitoring worm control in horses. Vet Parasitol. 2008;151(2-4):249-55.

9. Mekonnen Z, Meka S, Ayana M, Bogers J, Vercruysse J, Levecke B, Comparison of individual and pooled stool samples for the assessment of soil-transmitted helminth infection intensity and drug efficacy. PLoS Negl Trop Dis. 2013;7(5):e2189.

10. Kure A, Mekonnen Z, Dana D, Bajiro M, Ayana M, Vercruysse J, et al. Comparison of individual and pooled stool samples for the assessment of intensity of Schistosoma mansoni and soil-transmitted helminth infections using Kato-Katz technique in southwest Ethiopia. Parasite Vectors. 2015;8(1):489. In Press.

11. Hotez PJ, Kamath A. Neglected tropical diseases in sub-saharan Africa: review of their prevalence, distribution, and disease burden. PLoS Negl Trop Dis. 2009;3(8):e412.

12. Deribew K, Tekeste Z, Petros B. Urinary schistosomiasis and malaria associated anemia in Ethiopia. Asian Pac J Trop Biomed. 2013;3(4):307-10.

13. Ayele B, Erko B, Legesse M, Hailu A, Medhin G. Evaluation of circulating cathodic antigen (CCA) strip for diagnosis of urinary schistosomiasis in Hassoba school children, Afar. Ethiopia Parasite. 2008;15:69-75.

14. World Health Organization. Basic Laboratory Methods in Medical Parasitology. Geneva: WHO; 1991.

15. Ibironke OA1, Phillips AE, Garba A, Lamine SM, Shiff C. Diagnosis of Schistosoma haematobium by detection of specific DNA fragments from filtered urine samples. Am J Trop Med Hyg. 2011;84(6):998-1001.

16. Knopp S, Corstjens PL, Koukounari A, Cercamondi Cl, Ame SM, Ali SM, et al. Sensitivity and specificity of a urine circulating anodic antigen test for the diagnosis of Schistosoma haematobium in low endemic settings. PLoS Negl Trop Dis. 2015;9(5):e0003752

17. Levecke B, Anderson R, Berkvens D, Charlier J, Devleeschauwer B, Speybroek N, et al. Mathematical inference on helminth egg counts in stool and its applications in mass drug administration programmes to control soil-transmitted helminthiasis in public health. Adv Parasitol. 2015;87:193-247.

18. Speybroeck N, Williams C, Lafia K, Devleesschauwer B, Berkvens D. Estimating the prevalence of infections in vector populations using pools of samples. Med Vet Entomol. 2012;26:361-71.

19. Mitchell S, Pagano M. Pooled Testing for Effective Estimation of the Prevalence of Schistosoma mansoni. Am J Trop Med Hyg. 2012;87(5):850-61.

\section{Submit your next manuscript to BioMed Central and take full advantage of:}

- Convenient online submission

- Thorough peer review

- No space constraints or color figure charges

- Immediate publication on acceptance

- Inclusion in PubMed, CAS, Scopus and Google Scholar

- Research which is freely available for redistribution 